\title{
Soundscapes, human restoration and quality of life
}

\author{
Irene VAN KAMP ${ }^{1}$, Elise VAN KEMPEN ${ }^{1}$, Ronny KLAEBOE ${ }^{2}$, Hanneke KRUIZE ${ }^{1}$, A. L. BROWN ${ }^{3}$ \\ and Peter LERCHER ${ }^{4}$ \\ ${ }^{1}$ Nat. Inst. Public Health and the Environment, Netherland \\ ${ }^{2}$ Institute of Transport Economics, Oslo, Norway \\ ${ }^{3}$ Griffith University, Brisbane, Australia \\ ${ }^{4}$ Medizinische Universität, Innsbruch, Austria
}

\begin{abstract}
There is increasing interest in the notion that acoustic environments may affect human health, not only at high exposure levels, but also where the acoustic environment is pleasant and of high quality. In this paper we explore the notion that access to high quality acoustic environments may have a role in quality of life. This can be intrinsically, or by way of mediation for people otherwise exposed to adverse environments, through some restorative and health promoting mechanism(s). First the limited literature on soundscapes and restoration, and their interconnection, is discussed. Next, the results of secondary analysis on several data sets are presented. It is concluded that evidence on the intrinsic positive value of areas with high acoustic quality (green-blue areas) for restoration, by way of an immediate pathway, is limited. Temporary respite from environmental noise can mitigate the negative effects on health and wellbeing, but the long-term effects have scarcely been studied. Based on the secondary analysis, key determinants of perceived soundscapes and overall evaluation of sound quality in different conditions are presented. Next, the evaluation of overall sound quality and perceived soundscapes is linked to restoration at places which people frequent to relax, socialize or for physical exercise.
\end{abstract}

Keywords: Soundscape, restoration, quality of life, wellbeing

I-INCE Classification of Subjects Number(s): 56.3, Soundscapes 51.4, 62.5

\section{INTRODUCTION}

Worldwide there is a revival of interest in the positive effect of landscapes, green and blue space, open countryside on human well-being, quality of life, and health, especially for urban dwellers. However, most studies do not account for the influence of the acoustic environment in natural environments either in a negative or positive way. One of the few studies in the field, which was performed by Kang and Zhang (1), identified relaxation, communication, dynamics and spatiality as the key factors in the evaluation of urban soundscapes. Remarkable is their finding that the general public and urban designers clearly value public space very different. The latter reported a much stronger preference for natural sounds and green spaces than the lay-observers. Do we, as professionals, tend to exaggerate the value of green and what characteristics of urban green space are key to health, wellbeing and restoration? And especially, what role does the acoustic quality and accompanying social quality play in all this? There is evidence that green or natural areas/wilderness/ or urban environments with natural elements as well as areas with a high sound quality can intrinsically provide restoration through spending time there. Also, merely the knowledge that such quiet and green places are available seems to work as a buffer effect between stress and health(2).

The key concept of restoration was first introduced by Kaplan \& Kaplan (3) in their Attention

\footnotetext{
1 Irene.van.kamp@rivm.nl, elise.van.kempen@rivm.nl, Hanneke.kruize@rivm.nl

2 Ronny.Klaeboe@toi.no

3 Lex.Brown@griffith.edu.au

4 Peter.Lercher@i-med.ac.at
} 
Restoration Theory (ART). Research into the mechanism confirmed that people can concentrate better after spending time in nature, or after looking at scenes of nature (3) (4). There is some indication that certain places contribute to restoration. Often it is assumed that this is related to aspects of quiet and green, but evidence on this combined effect of noise and green is still highly anecdotal and primarily focused on recreational areas outside cities rather than urban areas (2). Based on the available evidence we assume at least two mechanisms : 1) by an immediate pathway and 2) an indirect pathway by way of mediation or moderation where features of the immediate, and broader environment in which people live determine the effect of aversive features such as a lack of green and/or quiet (5) (6)

So far only a handful of studies is available addressing this issue. Very few studies have evaluated the physical and spatial characteristics of areas with good sound quality (7) (8). Booi and van den Berg (8) identified, by means of interviews, several features characterizing quiet areas, such as the presence of green and water, maintenance, colors, a sense of space, and odors. Suburban residents characterized a quiet area differently than did city dwellers. Earlier, Pheasant and colleagues (9) (10) found that maximum sound pressure level (LAmax), and the percentage of natural features of a location, were key factors influencing perceived tranquility. Also of interest are studies addressing the intrinsic restorative quality of the built environment. Lindal and Hartig (11) for example showed in an experimental setting that attributes of buildings influenced people's judgments about the potential to be beneficial for stress reduction.

In an early study of Ulrich et al (12) including physiologic measures, it was found that exposure to "pure" nature sites resulted in a slightly better restoration when compared with urban restoration sites, and that high levels of traffic noise was associated with poor recovery. The differences were small, but the trends are clear. Besides potential immediate restorative effects, there may be long-term effects of access to environmental amenities in the immediate living environment In general there are four mechanisms assumed that lead from green and tranquil space to health: via physical activity, via social interactions and relaxation and finally via reduced levels of traffic related air and noise pollution (13). The constraint of restoration has more recently been distinguished as a contributor to chronic stress that may be attributed to environmental conditions that undermine opportunities for restoration (14) (15).

This paper explores the role of sound in the process which leads from access to and use of green space to restoration and health. Results of a systematic review of the literature are combined with secondary analysis on two existing data sets. The main aim is first to explore the association between people's perceptions of the sound quality in different conditions (in hand near the home and at their favorite green area) using non parametric methods for analysis. Secondly, the aim is to study the association between perceived sound quality with several indicators of restoration and subjective health. This is done while accounting for demographic and contextual features in particular aspects of green (objective as well as subjective indicators), and social features (social cohesion), using multi-level regression.

\section{METHOD}

\subsection{Literature Review}

In the framework of a COST project on soundscapes a systematic literature search was performed for the specific association between soundscapes and health and wellbeing (2). Scopus databases were searched to identify relevant peer-reviewed English, German and Dutch studies published in the period between April 2000 and April 2013. A wide range of keywords was used related to tranquility, quiet, public or green space, restoration, stress and health. Several recent reviews, including both peer-reviewed and grey literature, were used to summarize the state of the art on the beneficial effects of green space on health and wellbeing, and the role of physical and social aspects in the association between sound/noise and wellbeing.

\subsection{TASTE (Towards a Sustainable Acoustic Environment)}

Data were derived from a study performed in the Netherlands in 2013, which was granted by the Strategic Program RIVM. Participants in this study were people of 18 years and older, recruited from 33 neighborhoods in three Dutch cities (Arnhem, Amsterdam and Rotterdam) which were later aggregated into 31 neighborhoods in view of equal size. Neighborhoods were selected according to their level of urbanization, and contrasting levels and variations in noise exposure, neighborhood lay-out, and were subsequently matched on socio-economic status. Per neighborhood, a random 
sample of 500 inhabitants (15,508 in total) was drawn from the municipal population registers of three cities. After two reminders, 3972 respondents returned the questionnaire which means a response rate of 26 percent. With the number of participants the target of at least 100 people per neighborhood was reached. This was defined in power analysis as a prerequisite to obtain a power of 85 and $90 \%$. For a detailed description of study design and methods we refer to Van Kempen et al.(7) (16).

The questionnaire included three sets of questions regarding perceived sound quality (i) The sounds people hear when a) in the dwelling, b)outdoor (within 5 meters from the dwelling) or c) while visiting their favorite place elsewhere in the city) was measured by asking how often they heard traffic sounds, human sounds, natural sounds, and other sounds. Answers were given on a 5-point scale ('never', 'occasionally', 'regularly', 'often', and 'very often'). (ii)The meaning of the sound that people perceive when a)in the dwelling, b) outdoor or c) visiting their favorite places elsewhere in the city was measured by an adapted version of the perceived soundscape questions developed by Axelsson, Nilsson and Berglund (17). This index was translated and back translated from Swedish to Dutch and back to Swedish in the framework of a previous study.

Respondents (iii) could indicate their overall evaluation of the sound situation on a 5-point category scale ("Very good", "good", "nor good nor bad", "bad”, or "very bad"). This was asked again for the three conditions of a) indoor, b) outdoor and c) at their favorite place elsewhere in the city. Information on both road traffic and cumulative noise levels (Yearly averaged LDEN, Lnight, L95night, L95day and variation in LDEN, variation in L95day, variation in L95day), was gathered at street level (Postcodal level) and neighborhood level. Geocoding at the level of dwelling was not an option since house numbers were not available. For neighborhood level exposures, the midpoint of the neighborhood was used by geocoding in GIS. In the NL this covers a street in general. Subsequently, modelled noise data available from national noise maps, with a resolution of $25 \times 25 \mathrm{~m}$ grids, were linked to the midpoint of these postal code areas and the midpoint of the neighborhood. Noise levels were assessed by means of the STAMINA model (Standard Model Instrumentation for Noise Assessment) (18).

Among others, the questionnaire contained an annoyance question measured by means of the standard ISO 11 point annoyance question index (19). In the analysis only the percentage highly annoyed was included following the Miedema standard, setting the cut-off point at 72 (20).

Questions regarding perceived neighborhood social cohesion, were derived from Dutch standard questions developed for the so called WoON questionnaire (21) and also described by Drukker et al. (22) and based on Sampson et al.(23).

Noise sensitivity was measured by means of an adapted version of the Weinstein noise sensitivity scale as was published and validated by Kishikawa, Matsui et al.(24).

Additional data at neighborhood level were derived from routine collected data regarding level of urbanization, demographic composition, greenness, amenities and by audits at neighborhood and street level (16).

\subsection{PHENOTYPE (Positive health effects of the natural outdoor environment in typical populations in different regions in Europe)}

The data used for secondary analysis are also derived from the Seventh Framework European study PHENOTYPE into the health effect of access and use of green area in four European cities of varying size in Spain, the UK, Netherlands and Lithuania. At the four study centers, people were selected from neighborhoods with varying levels of socioeconomic status and green and blue space (for details see 23). For this paper only data from the Dutch subsample were used.

Participants were people of 18 years and older, recruited from 30 neighborhoods in the city of Doetinchem, a provincial town with 56,832 inhabitants in the Eastern part of the Netherland with sufficient variation in types of green and blue space and levels of social economic status (SES).

Of the total 10,220 people between 18 and 75 years invited only 861 (8.4\%) agreed to participate in an oral interview. Non-response analysis in the Netherlands showed that responders value green/blue as more important for physical and social activities, for relaxation, and for commuting by foot or bike than people who decided not to participate. Again, because we deal with a stratified sample, the number of participants per neighborhood fulfils the criteria for obtaining statistical power.

For the basic green and blue measures in Doetinchem, the so called 'Top10NLwas used and generalized to conform to Urban Atlas land cover codes and minimum size criteria.

The questionnaire was constructed around the three mechanisms assumed to underlie the 
association between green/blue and health: social interaction, physical activity and a stress reducing mechanism. Per mechanisms, questions were asked about availability, use, importance of and satisfaction with green at different scale levels. Most questions were derived from existing and validated indices, some tailored to the specific objectives of PHENOTYPE. In the latter case questions were developed in English and translated and back translated in Dutch, Spanish, Catalan and Lithuanian where needed. Natural environments were defined as all public and private open spaces that contain "green" and "blue" natural elements such as street trees, forests, city parks and natural parks/reserves, including all types of water bodies, further referred to as "green spaces".

The questionnaire included two sets of questions regarding perceived sound quality comparable with the one described under 2.2 using 8 items instead of 12 items. Previous analysis confirmed earlier findings on the structure in two factors referring to a positive and negative dimension. Also, respondents could indicate their overall evaluation of the sound situation on a 5-point category scale ("very good", "good”, "nor good nor bad”, "bad”, or "very bad”). This was asked again for one condition only: their favorite place elsewhere in the city.

Questions regarding use of green space are derived from the UK Progress Survey (26) and were asked in relation to three scale levels: i) close to home (less than 15 minutes by foot or bike), ii) in the city or town (more than 15 minutes by foot or bike) and iii) in the vicinity of the city or town".

The restoration scale is an index developed by Korpela et al (27) (28) with 9 questions informing about the emotion people feel during or after a visit to their favorite green/blue space (e.g. During after a visit I feel: calmer, my concentration and alertness clearly increases, I get new enthusiasm and energy for my every day routine, I feel restored and relaxed). These questions could be answered with 5 categories ranging from "Fully fits" to "Does not fit at all" coded 1-5.

Questions regarding perceived neighborhood social cohesion were identical to what was used in the TASTE project.

Medically Unexplained Symptoms were measured by means of an adapted version of the 4DKL somatization Terluin (30). "In the past week how often have you suffered from: headache, muscle aches, nausea" The added items pertain to pain in back of head and fatigue. "They could be answered with 5 categories ranging from "Fully agree" to "Do not agree at all" coded $0-4$. The items were recoded and summed as prescribed by the guidelines.

Stress was measured by a newly composed indicator including questions on fatigue, medication use as a coping strategy, pain in back of head and two item from the MH5 scale (31) regarding feeling nervousness and feeling calm.

Socio-demographic characteristics: Individual-level covariates were obtained by using standardized questions about gender, age (in years), household composition (living alone/with partner and children younger than 12 years/ living alone or with partner and with or without older children), level of education (three categories: primary school or no education; secondary school/further education; university degree or higher), subjective income situation (three categories: "cannot make ends meet"; "just have enough to get along" and "being comfortable") and work status (employed/unemployed) operationalized in working at least 20 hours per week (according to CBS standard).

Multilevel regression analyses were applied to take into account the clustering of the data. Level 1 represented the individuals and Level 2 neighborhood. The same set of the following potential confounders was used in all models: gender, age, level of education, perceived income, employment status, household composition with/without children younger than 12 years and city (dichotomized) . Analysis was performed on the pooled data and the separate data and conducted using SPSS 22.0.

\section{RESULTS}

\subsection{Main Findings Literature Review}

Based on the available literature two types of restoration were discerned: Type 1 restoration refers to a high quality acoustic environment intrinsically providing restoration by way of an immediate pathway; Type 2 restoration refers to the effect of availability (knowledge) of a high (better) quality acoustic environment to a person who otherwise is subject to adverse effects of noise (at home). The latter includes availability of a quiet side in an otherwise high noise exposure dwelling, or access to/knowledge about nearby green areas. Both can be regarded as providing restoration by way of mediation.

Evidence on the intrinsic positive effect of areas with high acoustic quality (type 1) is limited. 
Studies on the effect of access to a quiet area at home as well as near the home (type 2) show that temporary respite of noise has beneficial health effect, but whether this holds long term as well is unclear. Also since most studies are cross sectional no firm conclusions can be drawn about the direction of the associations.

It is concluded that, in order to advance our understanding of the process by which these different mechanisms may operate, a more integrated approach is warranted-a contextual one in which the social and physical aspects are studied relating acoustic quality, via restoration, to health as was outlined by Lercher (29).

\subsection{Main Findings TASTE}

The main aim of the study addressed in this paper was: 1 . To investigate the determinants of people's perceptions of the sound quality a) in the dwelling, b) outdoor or c) visiting their favorite places elsewhere in the city near the homes. The relative importance of the determinants of perceived soundscape and overall acoustic quality was analyzed by means of non-parametric analysis. In a stepwise manner, the following models were tested:

Table 1: Steps in the non-parametric analysis .

\begin{tabular}{|c|}
\hline OUTCOMES: Soundscape \& Overall l Acoustic quality (three conditions) \\
\hline Predictors \\
\hline Level 1 City \\
\hline $\begin{array}{l}\text { Level } 2 \text { Neighbourhood (include urbanization/\% single homes and surface green and blue }+ \text { audit } \\
\text { (Business) activity, amenities Quality of public space, Amount of public green and blue (drop if } \\
\text { they do not add anything) }\end{array}$ \\
\hline Step 1: Delta night L95 night ,Delta day L95 day, Lden cummu, Lnight Cumu (Level 3) \\
\hline Step 2: Demographics (work, age , gender, education, type of household) \\
\hline Step 3: Perception of noise (road, neighbors, nature) \\
\hline Step 4: Annoyance Road, Neighbors, Mopeds \\
\hline Step 5: Social Cohesion, Noise sensitivity \\
\hline Step 6: Combination 1-5 \\
\hline
\end{tabular}

Overall it can be concluded that the parameters entered into the model, predict the overall sound quality (indoor, outdoor and at a favorite spot) better and with much less error than was the case in the prediction of the evaluation of the soundscape per condition. Associations between physical and social features at neighborhood, street, and individual level and perceived soundscapes and acoustic quality in general, was moderately strong, but significant as a consequence of a relatively large sample. People judge their indoor and immediate outdoor sound quality highly comparable, but outdoors traffic noise plays a key role and indoors the hearing of human sound and severe annoyance from neighbors. Next to the perception of and severe annoyance from different noise sources, social aspects of the neighborhood (attachment and cohesion) seem to play an important role. Noise indicators come on a second plan and do not add much to the prediction, nor do the audited features of the neighborhood (activity, the number of amenities, quality of public space and the amount of public green and blue space). Surprisingly, personal characteristics as noise sensitivity are also less important than one would expect based on previous studies focused on annoyance responses, where it showed that noise sensitivity was a very strong predictor of annoyance (32).

The perception of the sound quality at the most frequently visited area in the vicinity of peoples immediate environment are much less strongly associated with the included determinants and follow a different pattern all together. Although again the perceived noise sources as well as severe annoyance due to them are important predictors, social aspects are much less prominent than is the case for the 
evaluation of the sound quality in the vicinity and near the dwelling. Indicators for sound variance (Delta day and night) come forward as strong predictors. On the one hand it is thus confirmed that we need information about the physical and social context next to acoustic information in order to understand how people evaluate the acoustic quality at different scale levels. On the other hand the evaluation of the sound quality at places that people visit to recuperate from daily stress is highly dependent on the actual acoustics and in particular the variety in sound levels (7).

These findings confirm partly what we know from the scarce evidence on the theme. From the literature it was observed that in addition to the variation in time and spectral variance, also the spatial variation in sounds is assumed to influence peoples' perception of sound qualities(33) (34) (35). Human evaluation, according to Brown (36), of the sound quality at a certain spot depends less on absolute levels but more on the nature of the sounds that are presents and on the relative levels of those sounds.

\subsection{Main Findings PHENOTYPE (NL)}

The main aim of the analyses carried out in the context of this paper is to explore the association between people's perceptions of the sound quality of their favorite green area and several indicators of restoration and health. This is done while accounting for demographic and contextual features, in particular aspects of green (objective as well as subjective indicators), and social features (social cohesion), using multi-level regression analysis. While the main aim of TASTE was to study determinants of perceived acoustic quality, here we look at the predictive power of perceived soundscapes and perceived acoustic quality for perceived restoration and several health outcomes

The data were analyzed in three steps:

Table 2: Steps in the multilevel regression analysis

OUTCOMES: Restoration, Symptoms and Stress

Predictors

Level 1 Neighborhood

Step 1: Soundscape

Step 2: + Green indicators

Step 3: + Green indicators + Perceived Soundscape indicators (soundscape scales and general question)

Step 4: Full model adjusted for age, gender, education level, ethnicity, availability, use and importance of green, quality green. For Symptoms and stress also adjusted for ROS and social cohesion

Table 3 presents the results of the multilevel regression analyses for perceived restoration, symptoms and stress. Perceived restoration is predicted by a positive evaluation of the soundscape in the area people frequent for rest and recreation. This association remains significant after adjustment for available green at different distances from the dwelling and other relevant variables. The availability of green does not add to the prediction, but the total number of visits, the perceived quality and importance of green all contribute considerable to the prediction. Age is also an important predictor: younger people seem to profit more than people above 45.

Symptoms and stress-reactions show a reverse pattern in the sense that primarily the negative evaluation of the sound quality is an important predictor, also after adjusting for the availability of green. Access to green within 100 meters from the home is associated with more stress and more symptoms while available green within 300 meters from the home is associated with lower scores on both indices. Age, gender and level of education are associated with more symptoms and more stress. Importance of green, frequency of visiting green and the quality are not associated with stress and symptoms, but perceived social cohesion is. 

Table 3

: Mulitlevel regression analysis of soundscape questions on Restoration, Symptoms and stress in four models.

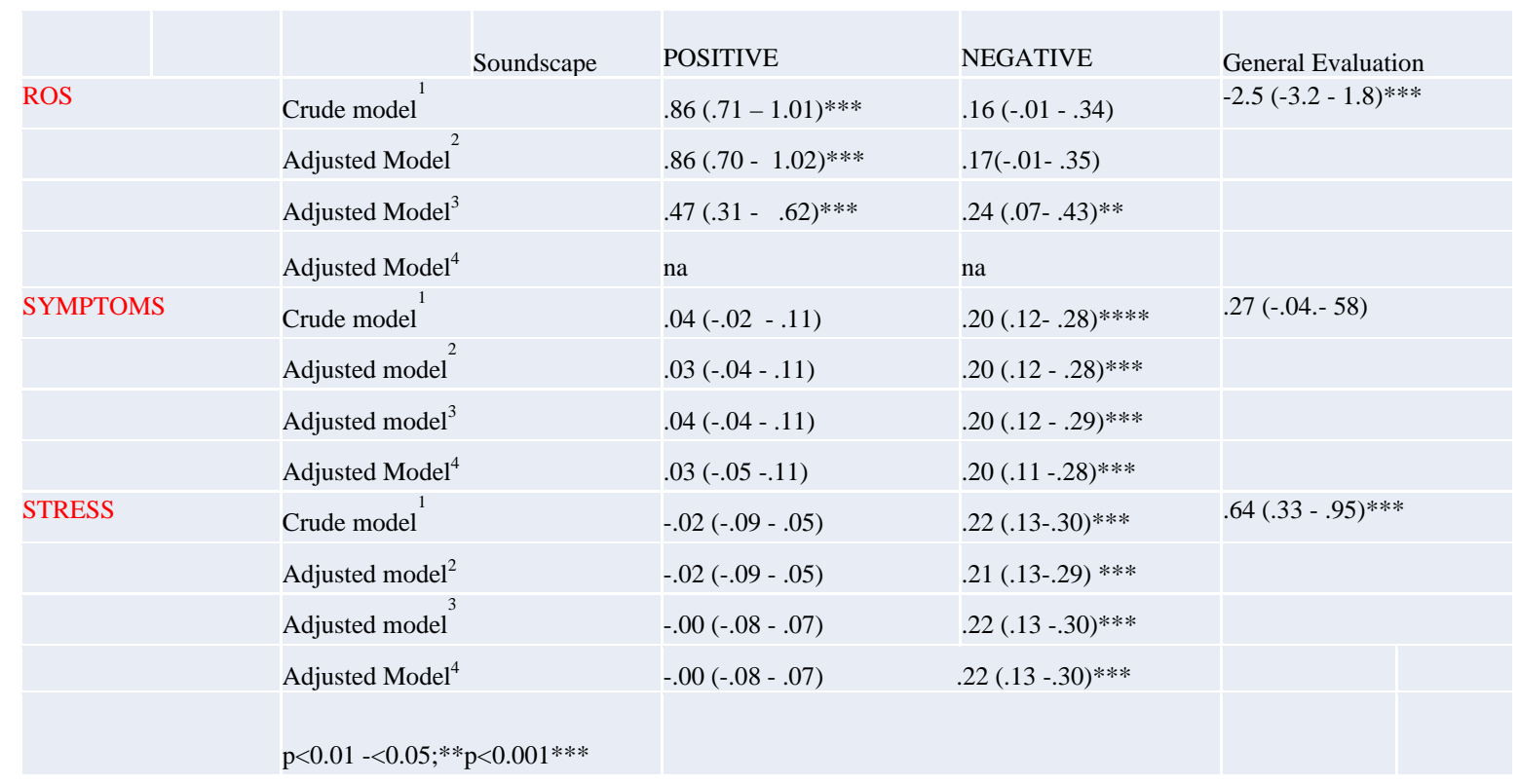

\footnotetext{
Doetinchem/NL

${ }^{1}$ Unadjusted model with a random intercept (neighborhood)

${ }^{2}$ Adjusted for green space indicators

${ }^{3}$ Adjusted for age, gender, education level, ethnicity, availability, use and importance of green, Quality Green,

Symptoms and Fatigue also adjusted for ROS

${ }^{4}$ Adjusted for age, gender, education level, ethnicity, availability, use and importance of green,

Quality Green,

Symptoms and Fatigue also adjusted for ROS and Social Cohesion
}

\section{CONCLUSIONS}

The literature review explored the notion that access to high quality acoustic environments may positively affect well-being, quality of life, and environmental health through some restorative or health and wellbeing promoting mechanism. Two types of restoration were discerned: Type 1 restoration refers to a high quality acoustic environment intrinsically providing restoration by way of an immediate pathway; Type 2 restoration refers to the effect of availability (knowledge) of a high (better) quality acoustic environment to a person who otherwise is subject to adverse effects of noise. The latter includes availability of a quiet side in an otherwise high noise exposure dwelling, or access to/knowledge about nearby green areas. Both can be regarded as providing restoration by way of mediation.

In order to verify these findings first people's perceptions of the sound quality of their immediate living environment in urban areas, and their determinants were analyzed. Results showed that soundscape perceptions differ per location and function/condition and are dependent on the physical and social contexts. While evaluations of the acoustic quality inside and outside the home are primarily determined by perceived noise sources and the level of annoyance, the evaluation of areas where people come for recreational purposes is primarily dependent on the type of noises people hear as well as a range of noise indicators including the average weighted noise levels, the noise variation and the peak levels of traffic noise. It seems hereby easier to predict peoples overall evaluations of the sound quality than their soundscape scores. The latter might be (со) determined by other variables, which were not included in this study. 
Secondly, on another dataset we studied the association between the soundscape perceptions with restoration, non-specific symptoms and indicators of stress while adjusting for demographic, physical and social aspects. Results show a consistent association between perceived soundscapes (positive and negative) and acoustic quality and symptoms, stress and its counterpart: restoration. Where perceived restoration is primarily associated with the importance, quality and use of green spaces near the home symptoms and stress show an immediate association with the availability of green near the home. Not clear is why the effect of green near the home and at 300 meters from the home show reverse patterns. Finally, perceived social cohesion is a very important predictor: a high level of social cohesion is associated with lower levels of stress and symptom report, after adjustment for other confounders including educational level etc.

For both studies the main limitation is that we are dealing with cross sectional data.

The results of detailed analysis show that people's judgement about their sound situation is dependent on context. Social aspects play a role in the indoor and outdoor condition, but not at the favorite spots which people visit most often. As expected, the indoor evaluation of the acoustic quality is more dominated by hearing human sounds and severe annoyance from neighbor noise, while the outdoor evaluation is predominantly determined by the perception of traffic sounds. Positive and negative soundscapes as well as the overall evaluation of the acoustic quality are important predictors of restoration, stress and symptoms also after adjustment for relevant aspects such as availability, use, quality and importance of green space, demographic features and social aspects.

These findings have important implications for the interpretation of previous studies, which were primarily focused on the negative aspects and annoyance only. When at the level of home we only focus on annoyance, we miss the influence of the broader social and physical context. At the level of recreational (green urban) areas where people go for relaxation the aspect of noise levels and in specific the variation in noise should receive more attention in future research. The interaction between physical, social and acoustic features is also relevant from a planning point of view. 


\section{ACKNOWLEDGEMENTS}

The results reported in this paper is based in research carried out in the framework of three different projects: the COST project Soundscape of European Cities and Landscapes (COST TD0804), the RIVM Strategic Programme (SPR nummer ), in which expertise and innovative projects prepare RIVM to respond to future issues in health and sustainability and thirdly European Community's Seventh Framework Programme (FP7/2007-2013) under grant agreement no: 282996 (ENV.2011.1.2.3-2) (Positive effects of natural environment for human health and well-being)

\section{REFERENCES}

1. Kang J, Zhang M (2010) Semantic differential analysis of the soundscape in urban open public spaces Building and Environment Volume 45, Issue 1, Pages 150-157.

2. Kamp Irene van, Ronny Klæboe, A. Lex Brown, and Peter Lercher (2015) Human Restoration, and Quality of Life Chapter 3 in Soundscape and the Built Environment edited by Jian Kang, Brigitte Schulte-Fortkamp CRC Press ISBN 9781482226317 - CAT\# K22479.

3. Kaplan R, Kaplan S. The Experience of Nature. Cambridge: Cambridge University Press; 1989.

4. Van den Berg, A. E., Koole, S. L. and Van der Wulp, N. Y. 2003. Environmental preference and restoration: (How) are they related? J. Environ. Psychol., 23: 135-146.

5. Wells, N. M., and Evans, G. W. 2003. Nearby nature a buffer of life stress among rural children. Environ. Behav., 35(3): 311-330.

6. Van den Berg, A. E., et al. 2010. Green space as a buffer between stressful life events and health. Social Sci. Med., 70(8): 1203-1210.

7. Kempen, E. van, Devilee, J., Swart, W., and van Kamp, I. 2014. Characterizing urban areas with good sound quality: Development of a research design. Noise Health, 2014, 16, 73, 380-387.

8. Booi, H., and van den Berg, F. 2012. Quiet areas and the need for quietness in Amsterdam. Int. J. Environ. Res. Public Health, 9: 1030-1050.

9. Pheasant, R. J., Fisher, M. N., Watts, G. R., Whitaker, D. J., and Horoshenkov, K. V. 2010. The importance of auditory-visual interaction in the construction of 'tranquil space'. J. Environ. Psychol., 30: 501-509.

10. Pheasant, R. J., Watts, G. R., and Horoshenkov, K. V. 2009. Validation of a tranquility rating prediction tool. Acta Acust. united Acust., 95: 1024-1031.

11. Lindal, P. J., and Hartig, T. 2013. Architectural variation, building height, and the restorative quality of urban residential streetscapes. J. Environ. Psychol., 33: 26-36.

12. Ulrich, R. S., et al. 1991. Stress recovery during exposure to natural and urban environments. J. Environ. Psychol., 11(3): 201-230.

13. Mari Murel Hanneke Kruize, Irene van Kamp, Jeroen P. van der Sluijs Mapping Salutogenic Benefits of Urban Green Spaces: A narrative Review of the Literature (submitted).

14. von Lindern, E., Hartig, T., \& Lercher, P. (2016). Traffic-related exposures, constrained restoration, and health in the residential context. Health \& Place, 39, 92-100.

15. Terry Hartig,Camilla Kylin and Gunn Johansson (2007) The Telework Tradeoff: Stress Mitigation vs. Constrained Restoration Applied Psychology, Volume 56, Issue 2, pages 231-253, April 2007

16. Devilee, Jeroen, Elise van Kempen, Wim Swart, Irene van Kamp and Caroline Ameling Assessment of spatial and physical neighborhood characteristics that influence sound quality and herewith well-being and health (submitted).

17. Axelsson, M., Nilsson, E., and Berglund, B. 2010. A principal components model of soundscape perception. J. Acoust. Soc. Am., 128(5): 2836-2846.

18. STAMINA - Model description. Standard Model Instrumentation for Noise Assessments Public on:2010-08-03, Schreurs EM, Jabben J, Verheijen RIVM Report 680740003

19. ISO/TS 15666:2003 Acoustics -- Assessment of noise annoyance by means of social and socio-acoustic surveys.

20. Miedema, ME, Oudshoorn CGM (2001) Environmental Health Perspectives; 109; 4: 409-416.

21. https://www.rijksoverheid.nl/onderwerpen/onderzoeken-over-bouwen-wonen-en-leefomgeving/inh oud/lopende-onderzoeken/woononderzoek-nederland-woon

22. Drukker M., Charles Kaplana,Frans Feron, Jim van Os (2003) Children's health-related quality of life, neighbourhood socio-economic deprivation and social capital. A contextual analysis. Social Science \& Medicine Volume 57, Issue 5, Pages 825-841. 
23. Sampson Robert J., Stephen W. Raudenbush, Felton Earls (1997) Neighborhoods and Violent Crime: A Multilevel Study of Collective Efficacy Science, Vol. 277 no. 5328 pp. 918-924

24. H Kishikawa, T Matsui, I Uchiyama, M Miyakawa, K Hiramatsu, SA Stansfeld (2006), The development of Weinstein's noise sensitivity scale Noise \& Health; 8: 154-160.

25. Mark J Nieuwenhuijsen, Hanneke Kruize, et al (2015) Positive health effects of the natural outdoor environment in typical populations in different regions in Europe (PHENOTYPE): a study programme protocol BMJ Open 2014;4:e004951 doi:10.1136/bmjopen-2014-004951

26. Gidlow C, Cochrane T, Davey RC, Smith G, Fairburn J (2010): Relative importance of physical and social aspects of perceived neighbourhood environment for self-reported health. Preventive Medicine, 51(2):157-63.

27. Korpela, Kalevi, and Terry Hartig. "Restorative qualities of favorite places." Journal of environmental psychology 16.3 (1996): 221-233.

28. Korpela, Kalevi M., et al. "Restorative experience and self-regulation in favorite places." Environment and behavior 33.4 (2001): 572-589.

29. Lercher, P. 2007. Environmental noise: A contextual public health perspective. In Noise and Its Effects, ed. L. Luxon and D. Prasher, 345-377. London: Wiley.

30. Terluin B, Van Marwijk HWJ, Adèr HJ, De Vet HCW, Penninx BWJH, Hermens MLM, Van Boeijen CA, Van Balkom AJLM, Van der Klink JJL, Stalman WAB. The Four-Dimensional Symptom Questionnaire (4DSQ): a validation study of a multidimensional self-report questionnaire to assess distress, depression, anxiety and somatization. BMC Psychiatry 2006; 6:34

31. Ware JE, Jr, Kosinski M, Bayliss MS, McHorney CA, Rogers WH, Reczek A. "Comparison of Methods for the Scoring and Statistical Analysis of SF-36 Health Profile and Summary Measures: Summary of Results from the Medical Outcomes Study.” Medical Care. 1995;33(4):264-79.

32. Daniel Shepherd, David Welch, Kim N. Dirks and Renata Mathews (2010) Exploring the Relationship between Noise Sensitivity, Annoyance and Health-Related Quality of Life in a Sample of Adults Exposed to Environmental Noise, Int J Environ Res Public Health. Oct; 7(10): 3579-3594.

33. Health Council of The Netherlands. Quiet areas and health. The Hague: Health Council of The Netherlands; 2006.

34. Klaeboe R, Engelien E, Steinnes M. Context sensitive noise impact mapping. Appl Acoust 2006;67:620-42.

35. Klaeboe R, Kolbenstvedt M, Fyhri A, Solber S. The impact of an adverse neighbourhood soundscape on road traffic noise annoyance. Acta Acust United Acust 2005;91:1039-50.

36. Brown AL. (2010) Soundscapes and environmental noise management. Noise Control Eng J 2010;58:493-500. 